\title{
The Agile Robotics for Industrial Automation Competition
}

\author{
William Harrison, Anthony Downs, Craig Schlenoff
}

- The Agile Robotics for Industrial Automation Competition (ARIAC) is an annual simulation-based competition initiated in 2017. The competition challenges teams to design industrial robotic system control code to function in a dynamic environment. Each team's system is faced with challenges such as dropped parts, which it must address, without operator intervention, while continuing to function.
$\mathrm{T}$ he Agile Robotics for Industrial Automation Competition (ARIAC) is an annual competition initiated in 2017. ARIAC is managed by the National Institute of Standards and Technology (NIST), ${ }^{1}$ with support from the Open Source Robotics Foundation (OSRF).

One of NIST's research areas has been the quantification of industrial robot intelligence through the definition of robot agility metrics and ontologies (NIST 2016). ARIAC was conceived to support this objective. The competition is designed to promote robot agility in industrial applications by utiliz- 


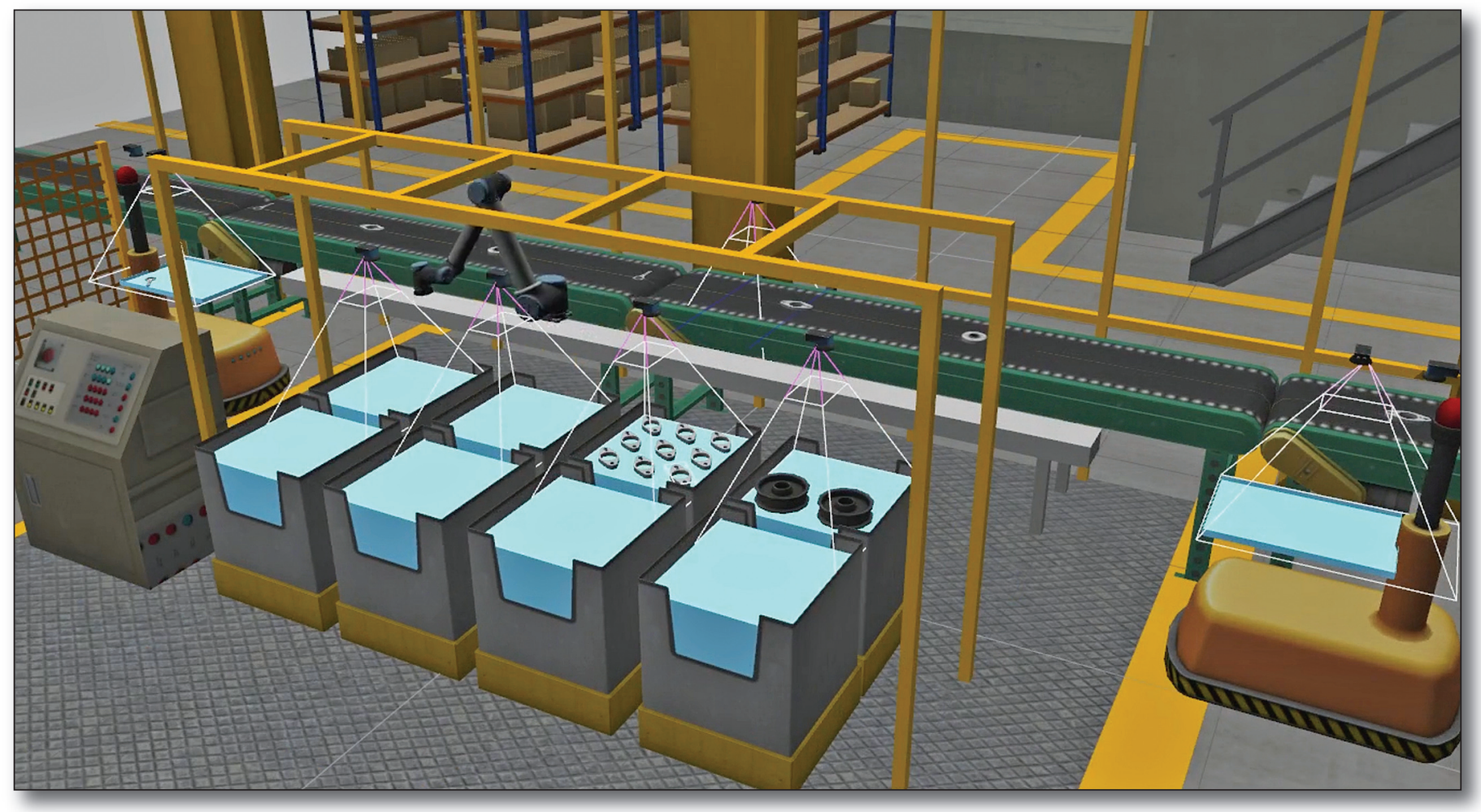

Figure 1: Example of the Simulation Environment.

ing the latest advances in artificial intelligence and robot planning. The goal of ARIAC is to solidify the field of robot agility, while also progressing the state of the art.

The organizers define industrial robot agility as a robotic system's (robot, controller, and sensors) ability to respond to a dynamic environment. This dynamic response includes handling errors like dropped parts or responding to changes in orders, all without operator intervention.

The competition addresses the aspect of robot agility that focuses on software, including knowledge representation, planning, and decision-making. While hardware aspects (such as different types of grippers) can play a large role in agility, they are not the focus of this competition. Perception and grasping have played a minimal role in the first year of the competition, but are expected to increase in importance in future years.

The competition ${ }^{2}$ was held completely in simulation using the Gazebo Robot Simulator. Gazebo is an open source Linux-based simulation environment that works very closely with the robot operating system (ROS). ${ }^{3}$ Gazebo ${ }^{4}$ was chosen because it is commonly used in academia. Additionally, it is free and therefore no mandatory monetary investment is required.

Teams competed by submitting robot control code and a sensor configuration for a kitting operation, as shown in figure 1 . The organizers chose kitting because of its similarity to assembly. Unlike assembly, however, kitting does not require a high-fidelity physics engine. Teams were tasked with assembling a kit both from bins of stationary parts and from a moving conveyor. After the robotic system finished the kit, the kit was placed on an autonomous guided vehicle (AGV) and taken away.

Teams were faced with such challenges as forced dropped parts and in-process order changes. Each team's system had to address these challenges and attempt to finish the kit autonomously in real time. The scoring metrics used in the competition were based partially on the robot agility metrics developed by NIST (Downs, Harrison, and Schlenoff 2016). Competition scoring took into account whether the kit was completed (both quantitative and qualitative metrics), how fast the kit was completed, and the cost of the sensor configuration. Each team's system was given a cost based on the number and type of sensors used. Typically, sensors that gave more information were priced higher than sensors that gave less. The total cost of each team's configuration factored into their score, where cheaper configurations resulted in higher scores.

The competition was preceded by three qualification phases. To compete in the competition, each 
team had to complete at least one qualifier. Initially, over 60 teams registered, with 10 teams qualifying for the final competition.

All of the competing teams were able to complete some aspect of the finals. No team, however, was able to achieve a perfect score. Additonally, although the organizers intended for all teams to use planning or AI approaches, this did not prove to be the case. Of the four teams that submitted entries for the finals, one used neither a conventional planning nor an AI approach. This team performed an in-depth analysis of the scenario and its patterns, which allowed them to design a system with minimal sensors and thus boosted their score quite a bit. Future iterations of ARIAC will discourage an approach like this by making the environment harder to predict. The other teams all instituted planning approaches, where their robotic systems would receive sensor data and then plan based on the current state of the world.

The winners of ARIAC 2017 were asked to present at a workshop held at the 2017 International Conference on Intelligent Robots and Systems, where travel was provided for one member of each winning team. The workshop also included talks from industry and academe that addressed industrial robot agility, as well as the future of ARIAC.

ARIAC 2018 will have a completely new scenario and setting. The organizers are considering an order fulfillment scenario, where orders are submitted and filled by a team's robotic system. The 2018 competition is also expected to provide cash prizes for the first-, second-, and third-place teams. The next round of ARIAC will likely be held in May of 2018.

\section{Disclaimer}

Certain commercial/open source software, hardware, and tools are identified in this article in order to explain our research. Such identification does not imply recommendation of or endorsement by the authors or NIST, nor does it imply that the software tools identified are necessarily the best available for the purpose.

\section{Notes}

1. National Institute of Standards and Technology - Agile Robotics for Industrial Automation Competition, www.nist.gov/ariac.

2. Competition video results: vimeo.com/224134238.

3. The Robot Operating System, Open Source Robotics Foundation, www.ros.org/about-ros.

4. Gazebo, Open Source Robotics Foundation, www.openrobotics.org, gazebosim.org.

\section{References}

Downs, A.; Harrison, W.; and Schlenoff, C. 2016. Test Methods for Robot Agility in Manufacturing. Industrial Robot: An International Journal 43(5): 563-72.

National Institute of Standards and Technology. 2016. Canonical Robot Command Language (CRCL). Gaithersburg, MD: National Institute of Standards and Technology.

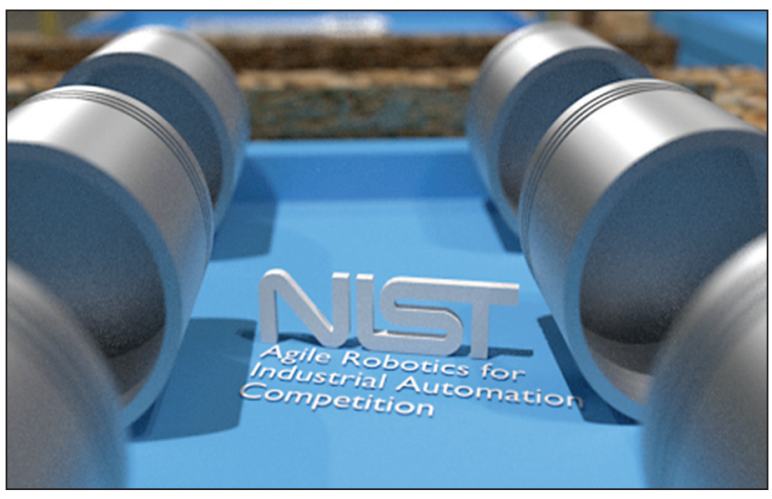

Anthony Downs is a mechanical engineer who has worked at NIST in the Intelligent Systems Division since 2005. He has worked on designing test methods for various projects, including Agility Performance for Robotic Systems, Collaborative Performance for Robotic Systems, and Emergency Response Robots, and on other list projects such as ARL UWB Testing, DARPA ASSIST, DARPA TransTac, DARPA TransApps, and DARPA BOLT. He has a BS in mechanical engineering from University of Maryland at College Park.

Craig Schlenoff is the associate program manager of the Robotics Systems for Smart Manufacturing Program, the project leader of the Agility Performance of Robotic Systems project, and the group leader of the Cognition and Collaboration Systems Group in the Intelligent Systems Division at the National Institute of Standards and Technology. His research interests focus on knowledge representation and ontologies, intention recognition, and performance evaluation of autonomous systems and industrial robotics. He previously served as the program manager for the Process Engineering Program at NIST and he is the director of ontologies at VerticalNet. He received his bachelor's degree from the University of Maryland, his master's from Rensselaer Polytechnic Institute, and his PhD from the University of Burgundy (France).

William S. Harrison III is a mechanical research engineer in the Department of Commerce's National Institute of Standards and Technology (NIST). Harrison's specialty within the project is virtual fusion, which is the mix of simulated and real components for process validation and training. His interests include virtual reality, game engines, augmented reality, and CG modeling. He received his bachelor's degree from the University of Michigan, his master's from the University of Florida, and his PhD from the University of Michigan. 